\title{
A cost analysis of Optical IP Switching in new generation optical networks
}

\author{
M. Ruffini, D. O'Mahony, L. Doyle \\ Centre for Telecommunication Value-Chain Research, \\ University of Dublin, \\ Trinity College, Dublin 2, Ireland
}

\begin{abstract}
In this paper we propose a simple model that analyzes the cost saving achieved by Optical IP Switching (OIS), a novel optical architecture, compared to a conventional IP-over-WDM network, where the IP router connects directly to the WDM module. The analysis we propose shows that OIS can bring substantial cost savings in the initial investment of network operators.
\end{abstract}

Keywords: Optical IP Switching, IP over WDM, cost analysis.

\section{Introduction}

Advances in WDM technology have started a revolution in optical architectures, producing new network structures with higher capacity, simpler structure, higher dynamicity and easier reconfigurability. Part of the research community strongly believes that future architecture will progressively reduce the number of layers between IP and WDM [1]. Following this view, ATM and Sonet layers will gradually disappear giving way to IP over WDM architectures. Other researchers instead [2] believe that maintaining a Sonet switching layer will reduce the network overall cost, due to the lower cost of switching fabric compared to a routing fabric.

In [3] and [4] we have introduced a novel architecture called Optical IP Switching, where a photonic switch connects the IP and WDM layers. The idea is based on the creation of a lightpath to bypass intermediate hops when an IP flow of significant size is recognized; instead of being created end-to-end, the path is locally generated and its extension proceeds in a distributed fashion. Optical paths traverse the switches transparently, with no electrical conversion and grooming possibility: in order to exploit the coarse optical bandwidth, large IP flows are detected and aggregated together before being transported over the dedicated optical paths.

In this paper we compare the Optical IP Switching (OIS) architecture to the IP-over-WDM model, from a cost perspective, using a simple model. In our calculation we only consider costs for initial investment (CAPEX); however we believe that intrinsic automatic traffic engineering capabilities of OIS will have a high impact also in reducing network operational costs (OPEX).

\section{Cost model}

We have produced models for two general network scenarios that may well represent most of the real network topologies: one considers a plain backbone network (we use 100 core nodes in our analysis [5]), the second instead considers a hierarchical structure where a core network interconnects different edge networks. In the following paragraphs we begin giving the details of the first model and we describe how the second model can be inferred from it. The results of the cost analysis, performed feeding real cost variables into the model, reports the cost saving capabilities of the OIS architecture.

\subsection{Backbone network}

The network we consider for this model is made up of 100 nodes $(N)$, with average degree of 3.4 (d) [5]. The network is capable of delivering " $T$ " Terabit per second of uniformly distributed traffic. The total network capacity "Tc" is calculated multiplying $T$ by the average number of IP hops "h", which can be calculated, as suggested in [5], from:

$$
h \cong \sqrt{\frac{(N-2)}{d-1}}
$$

while,

$$
T c=T \cdot h
$$

\subsubsection{IP over WDM architecture}

As we are only interested in average behavior, our model assumes that traffic is uniformly distributed among all the nodes, from which we can infer the router capacity needed at each router:

$$
C=\frac{T c}{N}(G b p s)
$$

which is accomplished using "Tc/N/Rh" routing port, each working at the higher rate (relatively to the router ports in the OIS model described in par. 2.1.2) of "Rh" Gbps.

The cost of each node is calculated by summing up the costs of the router chassis (which depends on the "Tc/N" value), the cost of the router line cards, and the cost of the DWDM multiplexers. The cost of the total network is obtained multiplying the cost per node by the number of nodes $\mathrm{N}$ in the network. All the optical transport costs are omitted because they are considered to be similar for both the architectures under analysis.

\subsubsection{Optical IP Switching architecture}

The distinctive feature of this architecture is the use of a photonic switch between the IP router and the DWDM multiplexers. This allows the router to create optical cutthrough paths and switch aggregates of "Elephant" flows directly in the optical domain. Elephant flows are large, long lived IP flows that carry most of today's Internet traffic. Recent studies show that, due to its heavy tailed traffic distribution, $0.02 \%$ of Internet flows carry almost $60 \%$ of data traffic [6]; other sources state that Elephant flows may carry over $85 \%$ of the all data [7]. In our analysis we have used an average value of $75 \%$. 
We take into consideration this behavior with the parameter "s", which indicates the fraction of traffic suitable for optical switching.

Considering then that data can be aggregated on a transparently switched path only at the source, the channel rate may not be optimally exploited: the parameter "e" defines this aggregation efficiency.

The creation of optical paths in OIS is distributed and locally evaluated: each path, initially created between three adjacent nodes, can be extended as a result of a local decision, which depends on the node resources and traffic characteristics. The average length "p" of each dedicated optical path is expressed as a fraction of the average number of IP hops $h$.

The cost model for OIS nodes is organized considering that " $\mathrm{s} \mathrm{T}^{\mathrm{N}}$ " of the throughput is transported by dedicated optical paths. The remaining part, carried by "mice" flows is instead routed at the IP layer and can be evaluated as:

$$
\text { routing }_{1}=\frac{h \cdot T \cdot(1-s)}{N}
$$

Each optical path works at a data rate equal to "e* $\mathrm{Rl}$ ", being "Rl" the line rate of lower rate router ports dedicated to dynamic optical paths. The number of optical paths will be in the average:

$$
\text { Paths }=\frac{T \cdot s}{e \cdot R l}
$$

Since optical paths are shorter than the average number of IP hops, each optical path also consumes a certain amount of routing resources. Moreover switched packets need to be routed into the proper optical path at the source node. From the first observation we find out that each flow needs routing resources equal to:

$$
\text { routing }_{2}=e \cdot R l \cdot(h-p)
$$

Then we must add the routing resources consumed by the source node ${ }^{1}$;

$$
\text { routing }_{3}=e \cdot R l
$$

Multiplying routing 2 and routing 3 by the number of paths and dividing by the number of nodes, we get the average values per node.

Summing up these routing values we can calculate the overall routing capacity needed at each node (needed to calculate the router chassis cost) and the number of line cards needed. We consider cards with higher rate ports (same type as in par. 2.1.1) for routing1 and routing2. For routing3 instead, in order to have finer granularity, we consider cards with lower rate ports.

The total routing capacity needed at each node is:

Rout $_{-}$cap $=$routing $_{1}+\frac{\text { routing }_{2} \cdot \text { Paths }}{N}+\frac{\text { routing }_{3} \cdot \text { Paths }}{N}$ (8)

The number of high rate ports needed at each node is:

\footnotetext{
${ }^{1}$ Routing resources are also consumed at the destination node. However this is taken into account in the cost analysis, where the rate of each routing port is considered both in the TX and RX directions.
}

$$
\text { Ports }_{h}=\frac{\text { routing }_{1}+\frac{\left(\text { routing }_{2} \cdot \text { Paths }\right)}{N}}{R h} \text { (9) }
$$

while the number of lower rate ports at each node is:

$$
\text { Ports }_{l}=\frac{\text { Paths }}{N}
$$

being each path originated by a lower rate port.

Finally we calculate the number of switch ports needed at each node:

$$
\text { Ports }_{\text {photo-switch }}=\frac{\text { Paths } \cdot p}{N}
$$

\subsection{Hierarchical network}

In case of a topology made up of a core network surrounded by several edge networks, the cost-model can be derived from the model previously discussed. All the calculations are performed separately for the two networks and the costs summed up at the end. For Optical IP Switching however we considered that dedicated paths are always originated and terminated at the edge node, while flowing transparently through the core network.

\subsection{Further considerations}

Most network operators use double amount of core routers that are necessary, in order to protect against failures. Since this, together with link protection increases the cost of both architectures in the same way we have omitted it from our model. Restoration after link or router failures is in both cases handled at the IP level. However Optical IP switching, with its capability of quickly creating optical cutthrough paths, can better absorb sudden increases in traffic after a network failure.

In the transport layer, we have considered some additional costs due to the fact that in the OIS architecture optical paths are notably longer. We have considered the cost for additional amplification and dispersion compensation at each node, plus the use of more expansive long range interfaces.

Another issue with OIS, like in any other all-optical architecture, is that wavelength contention can prevent the creation of new optical paths even if there is still capacity available in the network. The issue was not explicitly considered in the model, and is partly alleviated by the distributed and local control structure of OIS. Its effects however can be included by considering lower values for the "e" parameter, which increases the number of wavelengths needed to support a given amount of network traffic.

\section{Cost analysis}

Table 1 reports the cost variables we have used for our model. The cost for the IP over WDM network is the sum of the cost of the line cards, the router chassis and the multiplexer ports, multiplied by the number of nodes.

For the OIS network instead, besides routing associated costs, we need to consider the cost of the optical system: photonic switches, lower rate line cards, additional multiplexer ports and amplification and dispersion compensation at each node. 
Table 1. Cost variables used for the cost analysis.

\begin{tabular}{|l|l|l|}
\hline Devices & Cost & Sensitivity \\
\hline Mux/Demultiplexer (per port) & $\$ 400$ & -0.009 \\
\hline Line card (short range, per 10 Gbps port) & $\$ 157,000$ & \multirow{2}{*}{+0.07} \\
\hline Line card (long range, per 2.5 Gbps port) & $\$ 65,000$ & \\
\hline Amplification+dispersion compensation & $\$ 20,000$ & -0.01 \\
\hline Router chassis & See table & -0.007 \\
\hline Switch (per port) & See table & -0.044 \\
\hline
\end{tabular}

\begin{tabular}{|l|l|}
\hline \multicolumn{1}{|c|}{$\begin{array}{c}\text { Router } \\
\text { capacity } \\
\text { (Gbps) }\end{array}$} & $\begin{array}{c}\text { Chassis } \\
\text { cost }\end{array}$ \\
\hline$<160$ & $\$ 70,000$ \\
\hline $160-320$ & $\$ 130,000$ \\
\hline $320-640$ & $\$ 230,000$ \\
\hline $640-1280$ & $\$ 450,000$ \\
\hline$>1280$ & $\times \$ 450,000$ \\
\hline
\end{tabular}

\begin{tabular}{|c|c|}
\hline $\begin{array}{c}\text { Switch } \\
\text { dimension }\end{array}$ & $\begin{array}{c}\text { Per-port } \\
\text { cost }\end{array}$ \\
\hline$=<32 \times 32$ & $\$ 2,250$ \\
\hline$=<72 \times 72$ & $\$ 1,850$ \\
\hline$=<144 \times 144$ & $\$ 1,550$ \\
\hline$>144 \times 144$ & $\$ 1,550$ \\
\hline
\end{tabular}

Figure 1 shows how the cost difference between the two network architectures varies depending on the aggregation efficiency (e) and average path length ( $p$ ) parameter. In the figure the $y$ axis is expressed as the difference between average IP hops and average optical path length (h-p).

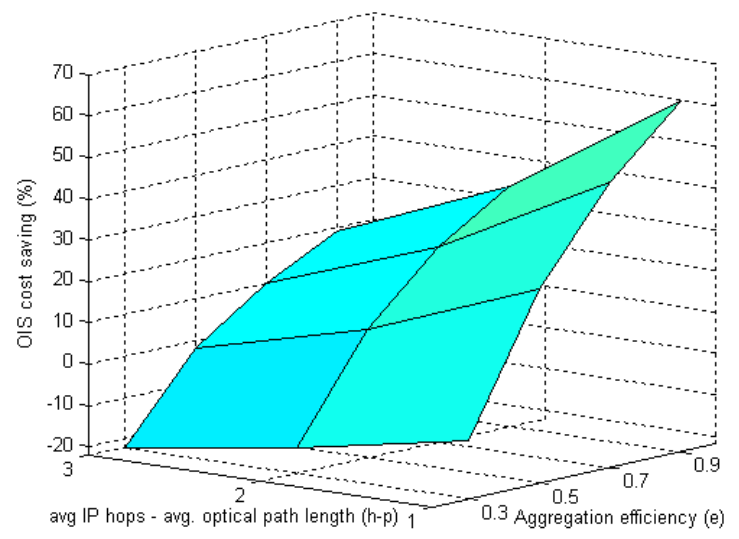

Figure 1: Cost savings of the OIS architecture compared to conventional IP-over-WDM (expressed in percentage).

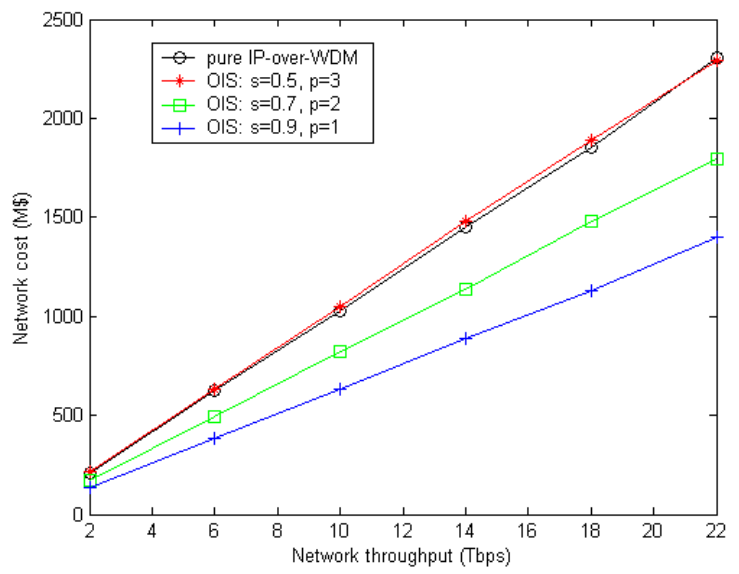

Figure 2 : Absolute cost values of the IP-over-WDM and OIS architectures.

We can observe that the OIS model has a cost advantage over the pure IP-over-WDM for values of $x$ higher than 0.5 , which can reach up to $63 \%$ when $e=0.9$ and $x=1$.
Figure 2 reports the absolute costs for the network architectures, for different values of the overall throughput $T$ and of the $p$ and $x$ parameters.

The sensitivity values on the right column of table 1 instead express how the OIS cost savings vary in percentage terms, when any of the equipment cost varies of $\pm 1 \%$ (they were calculated considering: $T=10$ Tbps, $\mathrm{e}=0.7$ and $h-p=2$ ). For example if we increase the cost of the demultiplexers by $1 \%$, the cost savings introduced by the OIS architecture decreases by $0.009 \%$.

If we apply the cost model to the hierarchical topology, considering the same number of average IP hops through the network, the OIS cost savings are very similar. However if we consider equal node degree the number of IP hops is lower in the hierarchical structure and the OIS cost savings become on average $15 \%$ less compared to the case previously described.

\section{Conclusions}

In this paper we have performed a cost analysis of a novel optical architecture, called Optical IP Switching, that uses photonic switches to create optical cut-through paths for aggregates of large IP flows.

We have developed a model that compares the cost of an OIS network to a conventional IP-over-WDM architecture. Results show that the architecture we propose can bring substantial savings under most operational conditions. This improvement derives from the capability of OIS to exploit the high level of correlation (in terms of sourcedestination pair) of Internet data. Instead of routing the traffic carried by large long lived flows with expensive routing hardware, OIS switches these flows in the optical domain using photonic switches characterized by a perport cost tens of times cheaper. The fact that most of Internet data is carried by these large flows (following a heavy tail distribution of the traffic), enhances the savings brought by the Optical IP switching architecture.

\section{Acknowledgment}

This material is based on works supported by Science Foundation Ireland under Grant No. 03/CE3/1405.

\section{References}

[1] S. S. Dixit: "IP over WDM", Chapter 6, p. 158, John Wiley \& Sons, 2003.

[2] S. Sengupta, V. Kumar, D. Saha: "Switched Optical Backbone for Cost-Effective Scalable Core IP Networks", IEEE Communications Magazine, vol. 41, no. 6, June 2003.

[3] Ruffini M., O'Mahony D., Doyle L.: "A Testbed Demonstrating Optical IP Switching (OIS) in Disaggregated Network Architectures", Proceedings of IEEE Tridentcom, Barcelona, Spain, March 1-3, 2006.

[4] G. Mulvihill, M. Ruffini, F. Smith, L. Barry, L. Doyle, D. O'Mahony: "Optical IP Switching a Solution to Dynamic Lightpath Establishment in Disaggregated Network Architectures", Proceedings of ICTON 06, Nottingham, UK, 18-22 Feb, 2006 (Invited paper).

[5] S. K. Korotky: "Network Global Expectation Model: a Statistical Formalism for Quickly Quantifying Networl Needs and Costs", IEEE journal of lightwave technology, vol.22, no. 3, March 2004.

[6] T. Mori, M. Uchida, R. Kawahara, J. Pan, S. Goto: "Identifying Elephant Flows Through Periodically Sampled Packets". Proceedings of IMC 04, Taormina, Sicily, Italy, Oct 2004.

[7] W. Fang, L. Peterson: "Inter-AS Traffic Patterns and Their Implications", Proceedings of the IEEE Globecomm, Brazil, December 1999 